\title{
Theoretical analysis of beamforming steering vector formulations for acoustic source localization
}

\author{
Gilles Chardon
}

Université Paris-Saclay, CNRS, CentraleSupélec, Laboratoire des signaux et systèmes, 91190, Gif-sur-Yvette, France

\begin{abstract}
In three-dimensional acoustic source localization, setting the steering vector is an important choice. Four steering vector formulations are frequently used. In the noiseless case, two of them yield a correct estimation of the position, the two others yielding correct estimation of the amplitude of the source, assuming its position is known. Here, noisy measurements are considered, and the steering vector formulations are compared in terms of bias and variance of the estimated position and amplitude, allowing a more informative assessment of their performances. An approximated value of the position bias of the biased formulations is given. Maximum likelihood estimation of the parameters of the source is derived, showing that it involves the combination of two steering vector formulations, for position estimation, followed by amplitude estimation. This analysis does not rely on the specific form of the Green function, and remains relevant for general propagation media.
\end{abstract}

Keywords: beamforming, source localization, maximum likelihood

\section{Introduction}

The beamforming method for acoustical sources localization and quantification of their power is one of the most popular acoustical imaging methods[1]. Based on the focusing of an array towards a direction of interest by delaying received signals (or, in the frequency domain, applying phase terms to the complex amplitudes of the signals), this method is simple and yields accurate results in settings where sources are well-separated. In particular, beamforming does not necessitate to tune parameters such as the number of expected sources or a regularization parameter, as in more complex methods such as subspace methods (MUSIC [2], ESPRIT [3], etc.) or sparsity based methods (Orthogonal Matching Pursuit [4], $\ell_{1}$-norm based methods [5], etc.).

In addition to its use as an imaging method, beamforming can also be used as a component of more sophisticated imaging methods aiming at better estimation of the parameters of the sources, in particular at lower frequencies. In Orthogonal Beamforming [6], beamforming is applied on the singular vectors of the spatial covariance matrix of the data. Iterative methods (e.g. CLEAN and CLEAN-SC [7]) can use beamforming as a selection step to add a new source to a set of identified sources. Also, deconvolution can be used to improve the resolution of beamforming maps, with the DAMAS algorithm [8] being one of the most famous examples of such methods (note however that the DAMAS algorithm was shown to solve the Covariance Matrix Fitting problem [9], which does not involve beamforming, and can be solved using more efficient numerical algorithms [10]).

\footnotetext{
Email address: gilles.chardon@centralesupelec.fr (Gilles Chardon)
}

We will consider here the case of frequency domain measurements, with one source. This simple case will allow to derive theoretical results. Two models can be used [11]: the conditional and unconditional models. The conditional model is used when sources emit sinusoidal signals. The parameters to be estimated are here the position and the complex amplitude of the source. The measurements are modeled as a random vector, with mean function of the parameters, and covariance depending on the power of the noise. Beamforming is applied by computing the scalar product $|\langle\mathbf{m}, \mathbf{h}(\mathbf{x})\rangle|^{2}$ between the measured data $\mathbf{m}$ and the steering vector $\mathbf{h}(\mathbf{x})$ for a set of positions $\mathbf{x}$ where the source is potentially located. In the unconditional model, the amplitude of the source is modeled by a random variable. Multiple snapshots are collected, and the spatial covariance matrix of the measurements is estimated by the sample covariance matrix $\hat{\Sigma}$. Then, the beamforming map is obtained by computing $\mathbf{h}(\mathbf{x})^{H} \hat{\Sigma} \mathbf{h}(\mathbf{x})$, in order to estimate the position and the power of the source. Measurement noise can be accounted for by subtracting the covariance matrix of the noise (usually assumed to be proportional to the identity matrix), or by diagonal removal. Here, we will assume that the covariance matrix of the noise is known, and use the former method.

Performances of source localization methods can be measured in multiple ways [12]. In the case of a unique source, performances can be assessed by considering the bias and variance of the estimation of the position of the source and of its amplitude in the conditional model, or its power in the unconditional model (in the rest of the text, "amplitude" will refer to amplitude or power, when relevant). For a scalar parameter $\theta$ to be estimated, the bias of an estimator $\hat{\theta}$ is defined as $b(\hat{\theta})=\mathrm{E}(\hat{\theta})-\theta$, (where $\mathrm{E}(\cdot)$ denotes the mathematical expectation), the difference between its expectation and the actual value 
of the parameter, and its variance as $\operatorname{Var}(\hat{\theta})=\mathrm{E}\left(|\hat{\theta}-\mathrm{E}(\hat{\theta})|^{2}\right)$. The mean squared error (MSE) of the estimator can be decomposed as $\operatorname{MSE}(\hat{\theta})=\mathrm{E}\left(|\hat{\theta}-\theta|^{2}\right)=b(\hat{\theta})^{2}+\operatorname{Var}(\hat{\theta})$.

For far-field sources, setting the steering vector is straightforward. However, in settings where the positions of the sources (and not only a direction of arrival) are to be estimated, and the field generated by a source cannot be modeled as a plane wave, the choice of the steering vector is not as obvious. Four different formulations of the steering vector were evaluated in [13]. It was found that formulations II and III were unbiased for the estimation of the power the source, and formulations I and IV were unbiased for the estimation of its position, with no formulation being unbiased for power and position estimation. However, measurement noise and variance of the estimations where not considered, preventing complete characterization of the performances of the steering vector formulations.

In this paper, a numerical and theoretical analysis of these four beamforming steering vectors is given. Measurement noise is considered, and bias and variance of the position and amplitude estimation are analyzed, allowing a more complete comparison of the steering vector formulations. In addition, the maximum likelihood estimator for the position and amplitude estimation is shown to involve two of the steering vector formulations (III and IV), allowing unbiased and efficient estimation of both position and amplitude. The code reproducing the figures is available online [14].

The article is structured as follows. Beamforming and the four steering vectors are recalled in section 2 . Power and amplitude estimation with known source position is considered in section 3 . Then, numerical results on position estimation errors are given in section 4. Position bias of formulations II and III is analyzed in section 5, and interpretation of the combination of formulations IV and III as the maximum likelihood estimator, and formulation I as a misspecified maximum likelihood estimator, of the parameters of the source is considered in section 6. Numerical results on amplitude estimation with unknown position are given in section 7 . Some remarks on beamforming with non-Gaussian models are given in section 8. Section 9 concludes the paper.

\subsection{Some notations}

The complex conjugate of a complex number $z$ is denoted $\bar{z}$. The conjugate transpose of a column vector $\mathbf{u}$ is indicated by $\mathbf{u}^{H}$. The scalar product of two complex vectors $\mathbf{u}$ and $\mathbf{v}$ of $\mathbf{C}^{N}$ is denoted

$$
\langle\mathbf{u}, \mathbf{v}\rangle=\sum_{n=1}^{N} u_{n} \bar{v}_{n} .
$$

Univariate and multivariate complex normal variables are all assumed to have a relation matrix equal to zero [15]. We recall that the complex normal vector of dimension $N$ with mean $\mu$ and covariance matrix $\Sigma$, denoted $C \mathcal{N}(\mu, \Sigma)$, has the density

$$
f(\mathbf{x})=\frac{1}{\pi^{N} \operatorname{det} \Sigma} \exp \left(-(\mathbf{x}-\mu)^{H} \Sigma^{-1}(\mathbf{x}-\mu)\right)
$$

\section{Beamforming and steering vectors}

We begin this section by recalling the beamforming method for far-field measurements. Here, the acoustical field $p(\mathbf{x})$ generated by a source and measured at a point $\mathbf{x}$ is assumed to be a plane wave

$$
p(\mathbf{x})=A \mathrm{e}^{\mathrm{i} \mathbf{k}(\alpha) \cdot \mathbf{x}}
$$

where $\mathbf{k}(\alpha)$ is a vector pointing from the direction $\alpha$ of the source, with length the wavenumber $k=2 \pi f / c$ ( $f$ being the frequency and $c$ the wave velocity) and $A$ is the complex amplitude of the source. Acoustical data are collected on an array of $N$ microphones. An array manifold $\mathbf{a}(\alpha)$ models the array geometry and wave propagation: its coefficients are the complex amplitudes of the acoustical field generated by a source of unit amplitude with direction of arrival $\alpha$ :

$$
a_{n}(\alpha)=\mathrm{e}^{\mathrm{i} \mathbf{k}(\alpha) \cdot \mathbf{x}_{n}}
$$

where $\mathbf{x}_{n}$ is the coordinate of the $n$-th microphone. Complex amplitudes of the noiseless soundfield collected on a microphone array can be arranged in a vector $\mathbf{p}=A \mathbf{a}(\alpha)$.

In the conditional model, the amplitude $A$ is assumed to be deterministic, and the measurements $\mathbf{m}$ are assumed to follow the distribution $\mathrm{CN}\left(\mathbf{p}, \sigma^{2} \mathbf{I}\right)$, modeling a white Gaussian noise of variance $\sigma^{2}$. Equivalently, the measurements can be decomposed as

$$
\mathbf{m}=A \mathbf{a}(\alpha)+\mathbf{n}
$$

where the noise $\mathbf{n}$ is a centered complex normal vector of covariance matrix $\sigma^{2} \mathbf{I}$.

In this model, beamforming estimates the direction of arrival $\alpha_{0}$ by computing the scalar product between the measurements $\mathbf{m}$ and the steering vector $\mathbf{h}(\alpha)=\mathbf{a}(\alpha) / N$

$$
\begin{aligned}
B_{C}(\alpha) & =|\langle\mathbf{m}, \mathbf{h}(\alpha)\rangle|^{2} \\
& =\frac{1}{N^{2}}\left|\sum_{n=1}^{N} \mathrm{e}^{-\mathrm{ik} \cdot \mathbf{x}_{n}} m_{n}\right|^{2}
\end{aligned}
$$

and selecting $\hat{\alpha}=\operatorname{argmax} B_{C}(\alpha)$. As $\|\mathbf{h}(\alpha)\|_{2}$ is constant, the Cauchy-Schwarz inequality guarantees that for noiseless measurements, $B_{C}(\alpha)$ is maximal when $\mathbf{p}$ and $\mathbf{h}(\alpha)$ are colinear, that is, $\alpha=\alpha_{0}$. The amplitude is estimated by

$$
\hat{A}=\langle\mathbf{m}, \mathbf{h}(\hat{\alpha})\rangle \text {. }
$$

In the unconditional model, the amplitude $A$ is assumed to be random, distributed according a complex centered normal distribution of variance $\rho$ the power of the source, assumed to be independent with respect to the noise. Here, the measurements are distributed according to a centered complex normal distribution, with covariance matrix $\Sigma=\rho \mathbf{a}(\alpha) \mathbf{a}(\alpha)^{H}+\sigma^{2} \mathbf{I}$. In this model, $S$ snapshots $\mathbf{m}_{s}$ are collected (in practice, the measured signals are analyzed by a short time Fourier transform), and the covariance matrix of the data is estimated using the sample covariance matrix

$$
\hat{\Sigma}=\frac{1}{S} \sum_{s=1}^{S} \mathbf{m}_{s} \mathbf{m}_{s}^{H}
$$




\section{I $\quad[16][17][18][19]$ \\ II [8] [16] [20] [4] [19] \\ III [12] [7] [6] [21] [22] [23] \\ IV [24] [25] [26] [27]}

Table 1: A sampling of the recent beamforming literature, classified according to the steering vector formulation(s) considered in the article.

and estimation of the direction of arrival is achieved by considering

$$
B_{U}(\alpha)=\mathbf{h}(\alpha)^{H} \hat{\Sigma} \mathbf{h}(\alpha)
$$

As above, when the theoretical covariance matrix is used in (10), $B_{U}(\alpha)$ is maximal at the actual position of the source. In noiseless cases, the power of the source can be estimated by $\hat{\rho}=B_{U}(\hat{\alpha})$. In the case of noisy measurements, power can be estimated by

$$
\hat{\rho}=\mathbf{h}(\hat{\alpha})^{H}\left(\hat{\Sigma}-\sigma^{2} \mathbf{I}\right) \mathbf{h}(\hat{\alpha})
$$

when the power of the noise is known (which will be the case in this paper), or by diagonal removal for unknown noise power.

Estimation of the position of a source in a region close to a sensor array is less straightforward, as the soundfield cannot be considered planar anymore. Following [13] and assuming free-field propagation, a reference point $\mathbf{x}_{0}$ is fixed, and the coefficients of the source vector $\mathbf{a}(\mathbf{x})$ take the form

$$
a_{n}(\mathbf{x})=\frac{r_{0}}{r_{n}} \mathrm{e}^{-\mathrm{i} k\left(r_{n}-r_{0}\right)}
$$

where $r_{0}=\left\|\mathbf{x}-\mathbf{x}_{0}\right\|_{2}$ and $r_{n}=\left\|\mathbf{x}-\mathbf{x}_{i}\right\|_{2}$ (dependency of $r_{0}$ and $r_{n}$ on $\mathbf{x}$ is left implicit in the notation for the sake of clarity). $A$ is the amplitude at the reference point $\mathbf{x}_{0}$. Amplitude of the source at a fixed distance $r_{\star}$ (e.g. $\left.1 \mathrm{~m}\right)$ is given by $A_{\star}=\left(r_{0} / r_{\star}\right) A$. This amplitude does not depend on the choice of the reference point, and is intrinsic to the source.

Compared to the far-field formulation (4), this source vector raises two challenges: the $\ell_{2}$ norm of $\mathbf{a}(\mathbf{x})$ is not constant with respect to the source position $\mathbf{x}$, and for a given position, absolute values of the coefficients $a_{n}(\mathbf{x})$ are not equal.

\subsection{Four steering vector formulations}

Several steering vectors have been proposed to account for this more complex model. We recall here the four steering vector formulations considered in [13]. As the references collected in Table 1 show, all four formulations are used in the recent literature of acoustical source localization.

Formulation I. When expressed in the frequency domain, delay and sum beamforming is achieved by compensating the phase of the coefficients of $\mathbf{a}$, with:

$$
h_{n}^{\mathrm{I}}(\mathbf{x})=\frac{1}{N} \frac{a_{n}(\mathbf{x})}{\left|a_{n}(\mathbf{x})\right|} .
$$

This steering vector is similar to the farfield steering vector, accounting for the non-planar wavefront.
Formulation II. Here, in addition to compensating the phases as above, amplitudes are also compensated:

$$
h_{n}^{\mathrm{II}}(\mathbf{x})=\frac{1}{N} \frac{a_{n}(\mathbf{x})}{\left|a_{n}(\mathbf{x})\right|^{2}} .
$$

Formulation III. In this formulation, the steering vector is obtained by dividing the source vector by its squared $\ell_{2}$ norm:

$$
\mathbf{h}^{\mathrm{III}}(\mathbf{x})=\frac{\mathbf{a}(\mathbf{x})}{\|\mathbf{a}(\mathbf{x})\|_{2}^{2}} .
$$

Formulation IV. Finally, formulation IV considers normalized steering vectors:

$$
\mathbf{h}^{\mathrm{IV}}(\mathbf{x})=\frac{1}{\sqrt{N}} \frac{\mathbf{a}(\mathbf{x})}{\|\mathbf{a}(\mathbf{x})\|_{2}}
$$

\section{Amplitude and power estimation with known position}

Assuming that the position of the source $\mathbf{x}_{s}$ is known, analysis of the estimated amplitude and power is straightforward, as the model is linear.

According to (8) and (5), in the conditional model the estimated amplitude at the reference point $\mathbf{x}_{0}$ is given by

$$
\hat{A}=A\left\langle\mathbf{a}\left(\mathbf{x}_{s}\right), \mathbf{h}\left(\mathbf{x}_{s}\right)\right\rangle+\left\langle\mathbf{n}, \mathbf{h}\left(\mathbf{x}_{s}\right)\right\rangle,
$$

where $\mathbf{h}$ is chosen from the four steering vectors introduced above.

Theorem 1. In the conditional model with known position, the estimated amplitude Âfollows the distribution

$$
\hat{A} \sim \mathcal{C N}\left(A\left\langle\mathbf{a}\left(\mathbf{x}_{s}\right), \mathbf{h}\left(\mathbf{x}_{s}\right)\right\rangle, \sigma^{2}\left\|\mathbf{h}\left(\mathbf{x}_{s}\right)\right\|_{2}^{2}\right) .
$$

Proof. This is a direct consequence of Eq. (17).

Values of $\left\langle\mathbf{a}\left(\mathbf{x}_{s}\right), \mathbf{h}\left(\mathbf{x}_{s}\right)\right\rangle$ and $\left\|\mathbf{h}\left(\mathbf{x}_{s}\right)\right\|_{2}^{2}$ are given in Table 2 for the four formulations. As was remarked in [13], formulations II and III yield unbiased amplitude estimations. However, the variance of formulation II is higher. Indeed, as $\left|\left\langle\mathbf{h}^{\mathrm{II}}\left(\mathbf{x}_{s}\right), \mathbf{h}^{\mathrm{III}}\left(\mathbf{x}_{s}\right)\right\rangle\right|=1 /\left\|\mathbf{a}\left(\mathbf{x}_{s}\right)\right\|_{2}^{2}=\left\|\mathbf{h}^{\mathrm{III}}\left(\mathbf{x}_{s}\right)\right\|_{2}^{2}$, and by CauchySchwarz inequality,

$$
\begin{aligned}
\left\|\mathbf{h}^{\mathrm{III}}\left(\mathbf{x}_{s}\right)\right\|_{2} & =\frac{\left|\left\langle\mathbf{h}^{\mathrm{II}}\left(\mathbf{x}_{s}\right), \mathbf{h}^{\mathrm{III}}\left(\mathbf{x}_{s}\right)\right\rangle\right|}{\left\|\mathbf{h}^{\mathrm{III}}\left(\mathbf{x}_{s}\right)\right\|_{2}} \\
& \leq\left\|\mathbf{h}^{\mathrm{II}}\left(\mathbf{x}_{s}\right)\right\|_{2} .
\end{aligned}
$$

In the unconditional model, the estimated power $\hat{p}$ is obtained using eq. (11).

Theorem 2. In the unconditional model with known position, the estimated power $\hat{p}$ has the following mean and variance:

$$
\begin{aligned}
\mathrm{E}(\hat{p}) & =\left|\left\langle\mathbf{a}\left(\mathbf{x}_{s}\right), \mathbf{h}\left(\mathbf{x}_{s}\right)\right\rangle\right|^{2} p \\
\operatorname{Var}(\hat{p}) & =\frac{1}{S}\left(\left|\left\langle\mathbf{a}\left(\mathbf{x}_{s}\right), \mathbf{h}\left(\mathbf{x}_{s}\right)\right\rangle\right|^{2} p+\left\|\mathbf{h}\left(\mathbf{x}_{s}\right)\right\|_{2}^{2} \sigma^{2}\right)^{2} .
\end{aligned}
$$

Proof. See Appendix A. 


\begin{tabular}{ccc} 
& $\left\langle\mathbf{a}\left(\mathbf{x}_{s}\right), \mathbf{h}\left(\mathbf{x}_{s}\right)\right\rangle$ & $\left\|\mathbf{h}\left(\mathbf{x}_{s}\right)\right\|_{2}^{2}$ \\
\hline I & $\frac{1}{N} \sum_{n=1}^{N} \frac{r_{0}}{r_{n}}$ & $\frac{1}{N}$ \\
II & 1 & $\frac{1}{N^{2}} \sum_{n=1}^{N} \frac{r_{n}^{2}}{r_{0}^{2}}$ \\
III & 1 & $\frac{1}{\sum_{n=1}^{N} r_{0}^{2} / r_{n}^{2}}$ \\
IV & $\sqrt{\frac{1}{N} \sum_{n=1}^{N} \frac{r_{0}^{2}}{r_{n}^{2}}}$ & $\frac{1}{N}$
\end{tabular}

Table 2: Values of $\left\langle\mathbf{a}\left(\mathbf{x}_{s}\right), \mathbf{h}\left(\mathbf{x}_{s}\right)\right\rangle$ and $\left\|\mathbf{h}\left(\mathbf{x}_{s}\right)\right\|_{2}^{2}$ for the four formulations.

Conclusions similar to the conditional model are reached here, with unbiased estimation for formulations II and III, and smaller variance for formulation III compared to formulation II.

As the bias of formulations I and IV is known, it is suggested in [13] to correct the estimation by dividing the estimated amplitude or power by $\left\langle\mathbf{a}\left(\mathbf{x}_{s}\right), \mathbf{h}\left(\mathbf{x}_{s}\right)\right\rangle$ or $\left|\left\langle\mathbf{a}\left(\mathbf{x}_{s}\right), \mathbf{h}\left(\mathbf{x}_{s}\right)\right\rangle\right|^{2}$, for the conditional and unconditional models respectively. For formulation IV, this is equivalent to using formulation III. Indeed,

$$
\frac{\mathbf{h}^{\mathrm{IV}}(\mathbf{x})}{\left\langle\mathbf{a}(\mathbf{x}), \mathbf{h}^{\mathrm{IV}}(\mathbf{x})\right\rangle}=\mathbf{h}^{\mathrm{III}}(\mathbf{x}) .
$$

For formulation I, the new steering vector $\mathbf{h}^{\mathrm{I} \prime}$ is given by

$$
h_{n}^{\mathrm{I} \prime}=\frac{1}{\sum_{n=1}^{N} \frac{r_{0}}{r_{n}}} \frac{a_{n}(\mathbf{x})}{\left|a_{n}(\mathbf{x})\right|},
$$

for which

$$
\left\|\mathbf{h}^{\mathrm{I}}\right\|_{2}^{2}=\frac{N}{\left(\sum_{n=1}^{N} \frac{r_{0}}{r_{n}}\right)^{2}}
$$

Applying the Cauchy-Schwarz inequality to the vectors $(1 / \sqrt{N}, \ldots, 1 / \sqrt{N})$ and $\left(r_{0} / r_{1}, \ldots, r_{0} / r_{n}, \ldots, r_{0} / r_{N}\right)$, we obtain that $\left\|\mathbf{h}^{\mathrm{I}}\left(\mathbf{x}_{0}\right)\right\|_{2}^{2} \geq\left\|\mathbf{h}^{\mathrm{III}}\left(\mathbf{x}_{0}\right)\right\|_{2}^{2}$, implying that the bias corrected formulation I has a larger variance than formulation III.

As a conclusion of this section, formulation III offers the best performances for amplitude and power estimation in the case of known source position.

\section{Position estimation: numerical simulations}

We now consider the estimation of the position of the source. In this section, simulations are used to highlight the behavior of the four formulations in particular settings in the conditional model. Bias and variance of the estimated positions are considered. First results are obtained by simulating a planar uniform rectangular array of $9 \times 9$ microphones of dimensions $1 \mathrm{~m} \times 1 \mathrm{~m}$ located in the plane $z=0 \mathrm{~m}$ (when unspecified, coordinates are given in meters). A source of amplitude $A=1 \mathrm{~Pa}$ is placed at coordinates $\mathbf{x}_{s}=(0,0.5,1.5)$, and two references points are considered: the center of the array $\mathbf{x}_{0}=(0,0,0)$, and the position of a microphone $\mathbf{x}_{0}^{\prime}=(0,-0.375,0)$. The source is searched in the domain with coordinates bounded by $-0.5 \leq x \leq 0.5,0 \leq y \leq 1$ and $0.5 \leq z \leq 2.5$. Geometry of the problem is plotted in Figure 1.

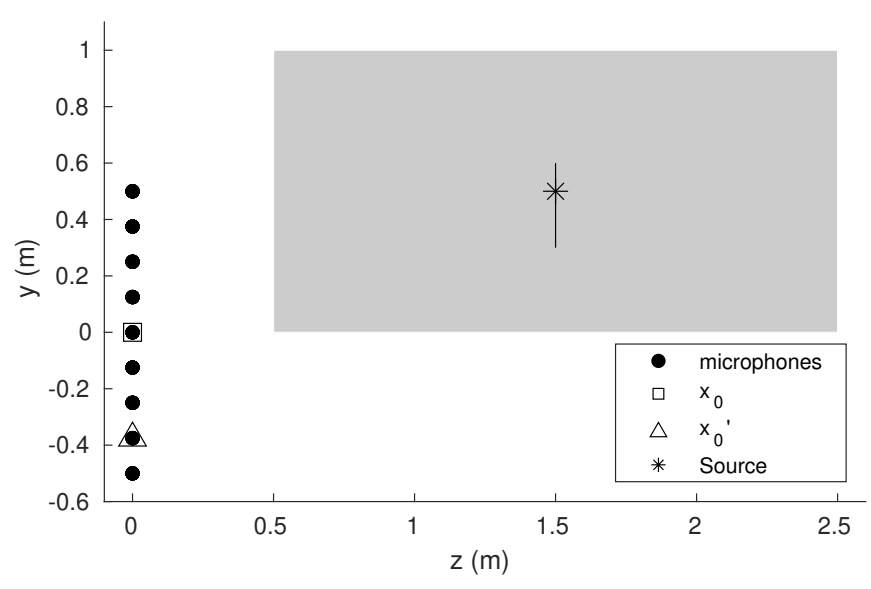

Figure 1: Geometry of the problem. Region of interest for Figure 2 indicated by the grey rectangle. The line on which the source is search in Figure 3 is indicated.

Bias. Beamforming maps are given on figure 2 for noiseless measurements at $k=15 \mathrm{~m}^{-1}$, with a discretization step of $5 \mathrm{~mm}$, in the plane $x=0$. As was shown in [13], formulations I and IV yield accurate positions, as the beamforming criterion is maximal at $\mathbf{x}_{s}$. For the reference point $\mathbf{x}_{0}$, formulations II and III exhibit estimated positions biased towards the array. Conversely, for reference point $\mathbf{x}_{0}^{\prime}$ (results denoted as II' and III'), estimated positions are biased away from the array. Formulations I and IV are unaffected by the choice of the reference point, as their definitions (13) and (16) ensure that $\mathbf{h}^{\mathrm{I}}$ and $\mathbf{h}^{\mathrm{IV}}$ remain invariant when the reference point changes (up to a phase term constant for all coefficients).

We note that formulations II and III are biased even when the coordinate $z$ is known. The criteria are plotted on figure 3 in function of the coordinate $y$, at $z=1.5 \mathrm{~m}$ and $k=5 \mathrm{~m}^{-1}$, for reference points $\mathbf{x}_{0}$ and $\mathbf{x}_{0}^{\prime}$. While the maxima of formulations I and IV are located at the actual $y$ coordinate of the source, the estimated position is biased for formulations II and III, with bias depending on the choice of the reference point.

Variance and MSE. To complete the analysis, we consider now noisy measurements with SNR $=10 \mathrm{~dB}$. Squared norm of the bias, variance and MSE are plotted on Fig. 4 in function of the frequency by averaging over 1000 realizations. The frequency axis is graduated using the Helmholtz number

$$
H_{e}=\frac{f d}{c}
$$

with $f$ the frequency, $d$ the aperture of the array, and $c$ the wave velocity. The beamforming criteria are maximized by gradient ascent, initialized by a grid search, and constrained to the region of interest.

Variances of all formulations are of the same order (for lower frequencies, estimated positions with formulations II and III are 

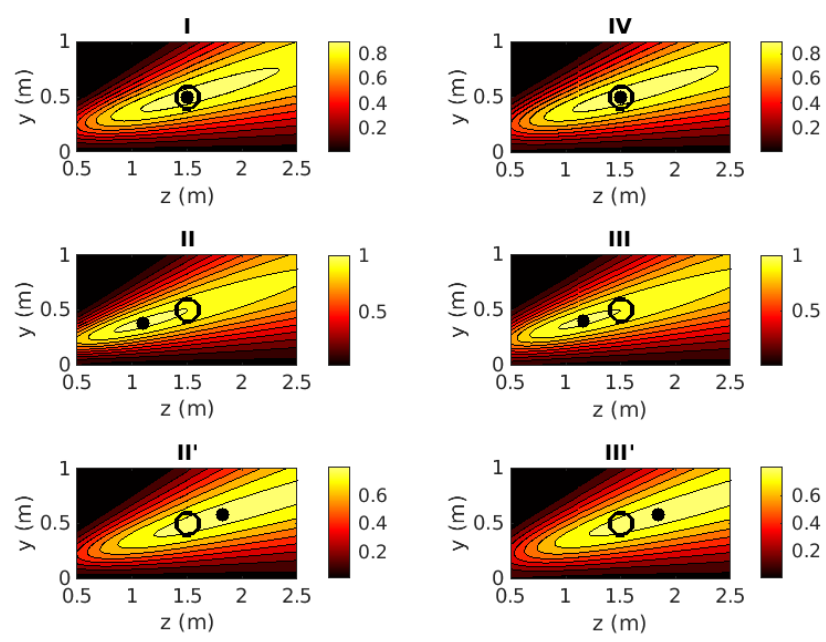

Figure 2: Value of the beamforming criterion for the four formulations (linear scale) in a planar region. For formulations II and III, results for reference points $\mathbf{x}_{0}$ (II and III) and $\mathbf{x}_{0}^{\prime}$ (II' and III') are given. Position of the source indicated by a circle, maximum value of the criterion by a disk.
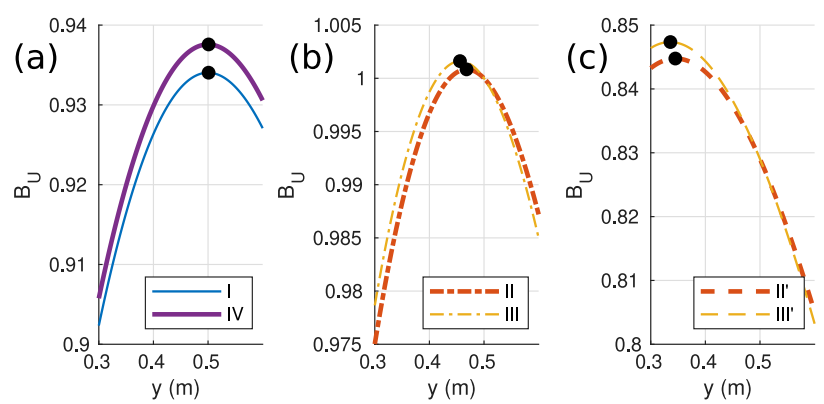

Figure 3: Value of the beamforming criterion for the four formulations (linear scale) on a line parallel to the array. (a) Formulations I and IV, (b) Formulations II and III, reference point $\mathbf{x}_{0}$, (c) Formulations II and III, reference point $\mathbf{x}_{0}^{\prime}$. Maximal values are indicated by disks (actual coordinate of the source $y_{s}=$ $0.5 \mathrm{~m})$ located on the boundary of the domain, which implies a lower variance). However, higher bias for formulations II and III imply higher MSE, for both reference points. Best performances are given by formulations I and IV which have here similar performances.

For a more precise comparison of formulations I and IV, a $3 \mathrm{D}$ array is considered, with 26 microphones placed at the vertices, edge midpoints and face centers of a cube of edge length $1 \mathrm{~m}$, centered at the origin. The source is placed at coordinate $(0.1,0.3,0.4)$, and MSE on Fig. 5 is plotted in function of the wavenumber $k$. The domain of interest is here the interior of the cube, and the reference point $\mathbf{x}_{0}$ is set at its center. Formulation IV appears to yield better performances than formulation I, with MSE halved at lower frequencies. The better performances of formulation IV are explained by the fact that it is capable of using the information contained in the relative amplitudes of the signals in addition to their phases.

As a conclusion, results of this section show that the estimation bias for the position of the source in formulations II and III depends on the position of the reference point, and that this bias also exists for two-dimensional problems. It is also shown that formulation IV has a smaller MSE compared to formulation I, in cases where the amplitudes of the signals yield information about the source position. The next two sections provide theoretical arguments supporting these observations.

\section{Approximation of the bias of Formulations II and III}

In this section, an approximate expression for the bias of formulations II and III is given, when the source to be localized is known to lie on a line. Measurements are here assumed noiseless. With $\mathbf{x}_{s}$ the position of the source and $\mathbf{u}$ a direction vector of the line where the source is searched, we define

$$
\begin{gathered}
B_{\mathrm{II}}(s)=\left|\left\langle\mathbf{a}\left(\mathbf{x}_{s}\right), \mathbf{h}^{\mathrm{II}}\left(\mathbf{x}_{s}+s \mathbf{u}\right)\right\rangle\right|^{2} \\
B_{\mathrm{III}}(s)=\left|\left\langle\mathbf{a}\left(\mathbf{x}_{s}\right), \mathbf{h}^{\mathrm{III}}\left(\mathbf{x}_{s}+s \mathbf{u}\right)\right\rangle\right|^{2}
\end{gathered}
$$

the value of the beamforming criterion on the line passing through $\mathbf{x}_{s}$, in function of the local coordinate $s$. When relevant, $B$ will be used to refer to both $B_{\mathrm{II}}$ and $B_{\mathrm{III}}$. As was remarked in [13], in general, the derivative of $B$ is nonzero at the actual position of the source, shifting the maximal value of the beamforming criterion away from the source.

In the neighborhood of the actual position of the source, $B(s)$ can be approximated by a quadratic Taylor polynomial

$$
B(s) \approx B(0)+B^{\prime}(0) s+\frac{1}{2} B^{\prime \prime}(0) s^{2}
$$

and an approximated value of the local coordinate $s^{\star}$ maximizing $B(s)$ is given by

$$
s^{\star} \approx-\frac{B^{\prime}(0)}{B^{\prime \prime}(0)}
$$

This can be interpreted as a step of the Newton optimization method [28]. 

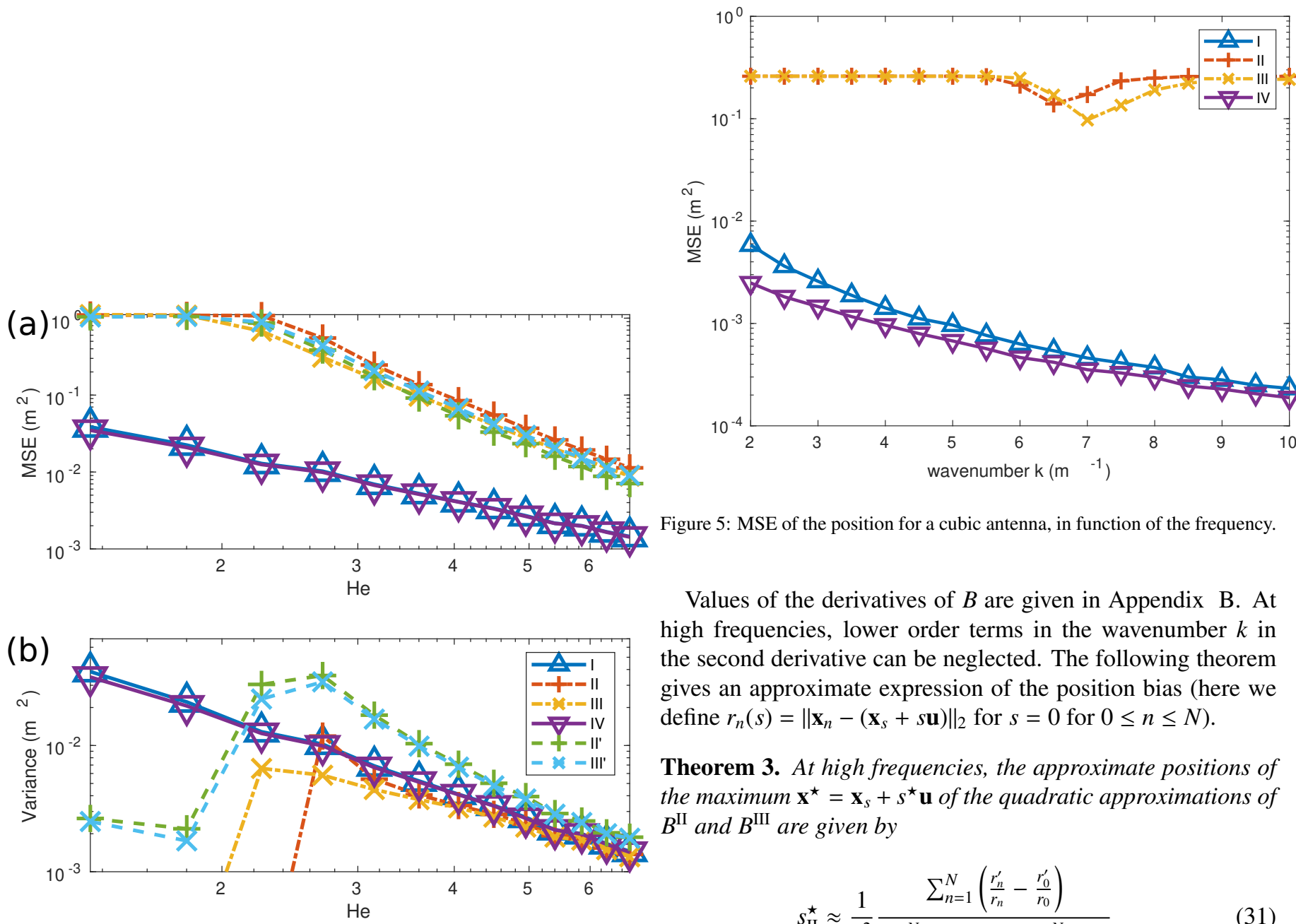

Values of the derivatives of $B$ are given in Appendix B. At high frequencies, lower order terms in the wavenumber $k$ in the second derivative can be neglected. The following theorem gives an approximate expression of the position bias (here we define $r_{n}(s)=\left\|\mathbf{x}_{n}-\left(\mathbf{x}_{s}+s \mathbf{u}\right)\right\|_{2}$ for $s=0$ for $\left.0 \leq n \leq N\right)$.

Theorem 3. At high frequencies, the approximate positions of the maximum $\mathbf{x}^{\star}=\mathbf{x}_{s}+s^{\star} \mathbf{u}$ of the quadratic approximations of $B^{\mathrm{II}}$ and $B^{\mathrm{III}}$ are given by

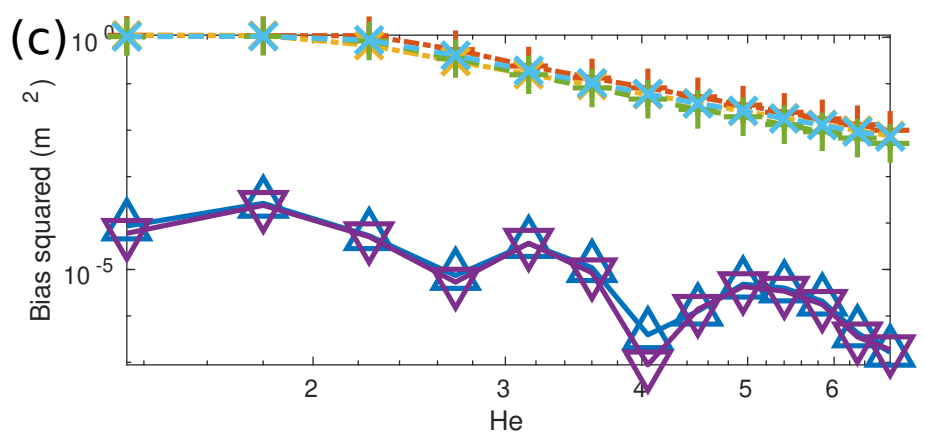

$$
\begin{aligned}
& s_{\mathrm{II}}^{\star} \approx \frac{1}{k^{2}} \frac{\sum_{n=1}^{N}\left(\frac{r_{n}^{\prime}}{r_{n}}-\frac{r_{0}^{\prime}}{r_{0}}\right)}{\left(\sum_{n=1}^{N} r_{n}^{\prime}\right)^{2} / N-\sum_{n=1}^{N} r_{n}^{\prime 2}} \\
& s_{\mathrm{III}}^{\star} \approx \frac{1}{k^{2}} \frac{\sum_{n=1}^{N} \frac{1}{r_{n}^{2}}\left(\frac{r_{n}^{\prime}}{r_{n}}-\frac{r_{0}^{\prime}}{r_{0}}\right)}{\left(\sum_{n=1}^{N} \frac{r_{n}^{\prime}}{r_{n}^{2}}\right)^{2} / N-\sum_{n=1}^{N}\left(\frac{r_{n}^{\prime}}{r_{n}^{2}}\right)^{2}} .
\end{aligned}
$$

where $r_{n}$ and $r_{n}^{\prime}$ are evaluated at $s=0$, for $0 \leq n \leq N$.

Proof. See Appendix B.

The approximated bias depends on the position of the source, the position of the reference point and the geometry of the array. Comparison of the approximated value of the bias of the estimation of the $z$ coordinate and values obtained through numerical experiments are given for the case of the geometry described in Fig. 6 for formulation II, with an array of 25 microphones, and reference point at the origin. Microphones are uniformly spaced in the $x y$ plane, with coordinates $z$ designed so that the approximation bias for a source at $z=4 \mathrm{~m}$ is zero (see Appendix B for details).

Values of the approximated and numerically obtained position bias for different positions of the source are plotted in figure 7, showing good accordance at high frequencies. As predicted, the estimation bias of the source at $4 \mathrm{~m}$ is zero. Estimated ranges when the source is placed closer to the array are biased towards the array. Conversely estimated range of the source 


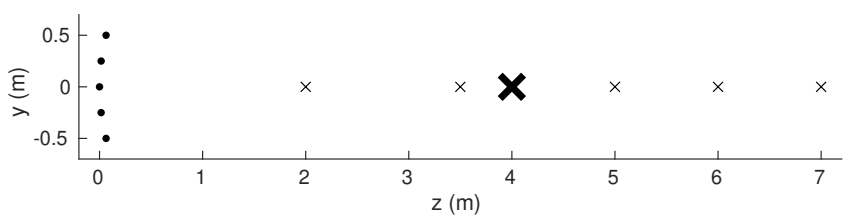

Figure 6: Geometry of the array ensuring unbiased range estimation of the source at $z=4 \mathrm{~m}$. Microphones in the plane $x=0$ indicated by disks, sources positions used in figure 7 by crosses.

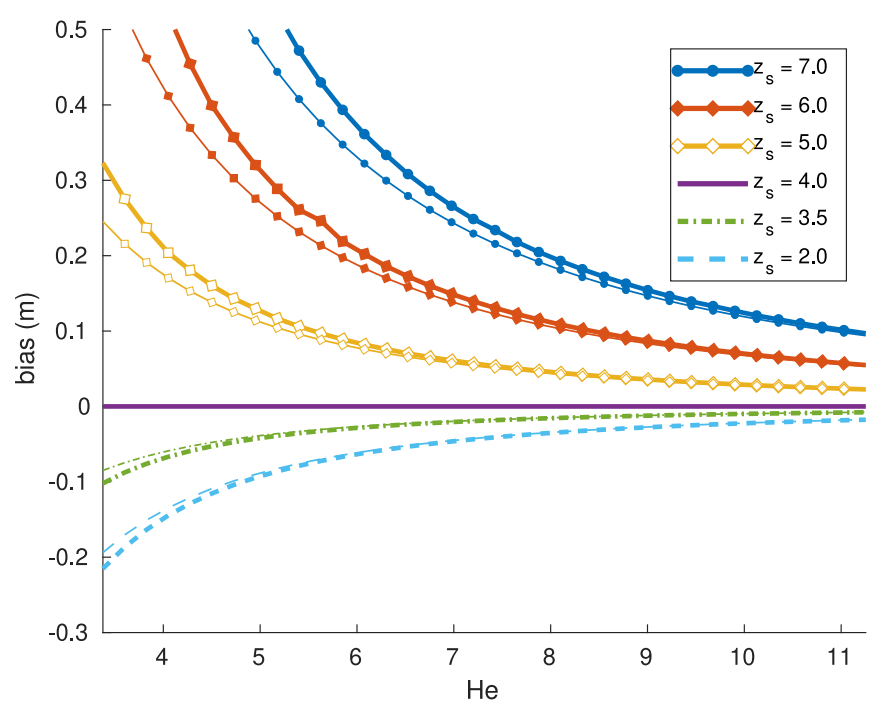

Figure 7: Position bias along coordinate $z$ in function of the frequency for formulation II. Thin lines: approximation (31), thick lines: numerical estimation.

when placed further from the array are positively biased. As remarked in [13], estimation bias decreases with increasing frequencies. Eqs. (31) and (32) show that this decay is quadratic with respect to the frequency.

The results of this section extend the observation of section 4 that the bias depends on the reference point. The bias also depends on the shape of the array and the position of the source. We remark that these results cannot be used for array design: unbiased range estimation for the source at $4 \mathrm{~m}$ was obtained with prior information on the position of the source, and in general does not guarantee unbiased estimation of the other coordinates.

\section{Maximum likelihood estimation}

Position estimation by formulations IV and I can be analyzed by interpreting them as a maximum likelihood estimator (MLE) [29], and a misspecified MLE (MMLE) [30] respectively.

The MLE is one of the most simple statistical estimators. Given measurements $\mathbf{m}$ following the probability distribution with density $f_{\theta}$, and $\theta$ the parameters to be estimated (here, $\theta$ contains the position $\mathbf{x}_{s}$ and amplitude $A$ or power $p$ of the source), the MLE estimate $\hat{\theta}_{\text {MLE }}$ is given by

$$
\hat{\theta}_{\mathrm{MLE}}=\underset{\theta}{\operatorname{argmax}} l_{\mathbf{m}}(\theta)
$$

where $l$ is the likelihood, defined by $l_{\mathbf{m}}(\theta)=f_{\theta}(\mathbf{m})$. The MLE has several desirable properties. Indeed, it is asymptotically unbiased, in the sense that its bias vanishes when SNR (for the conditional model [31]) or number of snapshots (for the unconditional model [11]) increase. Moreover, the MLE is asymptotically efficient, its variance reaches the Cramér-Rao lower bound under increasing SNR and number of snapshots, respectively.

Consequently, the MLE is often a good estimator.

Theorem 4. The MLE for the position and amplitude of a source in the conditional model is obtained in two steps, first by estimating the position (assumed to lie in a domain of interest $\Omega$ ) with

$$
\begin{aligned}
\hat{\mathbf{x}} & =\underset{\mathbf{x} \in \Omega}{\operatorname{argmax}}\left|\left\langle\mathbf{m}, \frac{\mathbf{a}(\mathbf{x})}{\|\mathbf{a}(\mathbf{x})\|_{2}}\right)\right|^{2} \\
& =\underset{\mathbf{x} \in \Omega}{\operatorname{argmax}}\left|\left\langle\mathbf{m}, \mathbf{h}^{\mathrm{IV}}(\mathbf{x})\right\rangle\right|^{2},
\end{aligned}
$$

followed by estimation of the amplitude using

$$
\begin{aligned}
\hat{A} & =\left\langle\mathbf{m}, \frac{\mathbf{a}(\hat{\mathbf{x}})}{\|\mathbf{a}(\hat{\mathbf{x}})\|_{2}^{2}}\right\rangle \\
& =\left\langle\mathbf{m}, \mathbf{h}^{\mathrm{III}}(\hat{\mathbf{x}})\right\rangle .
\end{aligned}
$$

Proof. See Appendix C.1.

We recognize, in these two steps, formulation IV for the position, and formulation III for the amplitude. As a consequence, formulation IV yields an asymptotically unbiased and efficient estimation of the source position. Combined with formulation III, an asymptotically unbiased and efficient estimation of the amplitude of the source is obtained.

In the unconditional model, the position of the source $\mathbf{x}_{s}$ and its power $p$ are estimated.

Theorem 5. With

$$
\begin{aligned}
B(\mathbf{x}) & =\frac{\mathbf{a}(\mathbf{x})^{H} \hat{\Sigma} \mathbf{a}(\mathbf{x})}{\|\mathbf{a}(\mathbf{x})\|_{2}^{2}} \\
& =\mathbf{h}^{\mathrm{IV}}(\mathbf{x})^{H} \hat{\Sigma} \mathbf{h}^{\mathrm{IV}}(\mathbf{x}),
\end{aligned}
$$

the MLE for the position of the source and its power in the unconditional model are given by

$$
\begin{aligned}
\hat{\mathbf{x}} & =\underset{\mathbf{x} \in \Omega}{\operatorname{argmax}} \frac{B(\mathbf{x})}{\sigma^{2}}-\log B(\mathbf{x}) \\
\hat{p} & =\frac{1}{\|\mathbf{a}(\hat{\mathbf{x}})\|_{2}^{2}}\left(B(\hat{\mathbf{x}})-\hat{\sigma}^{2}\right) \\
& =\mathbf{h}^{\mathrm{III}}(\hat{\mathbf{x}})^{H}\left(\hat{\Sigma}-\sigma^{2} \mathbf{I}\right) \mathbf{h}^{\mathrm{III}}(\hat{\mathbf{x}})
\end{aligned}
$$

Proof. See Appendix C.2 
MLE for the unconditional model also involves formulation IV for the estimation of the position, and formulation III for the estimation of the power. We note that (40) is not equivalent to maximizing $B(\mathbf{x})$, as the function $t \rightarrow t / \sigma^{2}-\log t$ is not increasing for all positive $t$. However, as the expectation of $\hat{\Sigma}$ is $\Sigma=\rho \mathbf{a}\left(\mathbf{x}_{s}\right) \mathbf{a}\left(\mathbf{x}_{s}\right)^{H}+\sigma^{2} \mathbf{I}$, values of $B(\mathbf{x})$ are likely to be larger than $\sigma^{2}$, for which the function is increasing.

We note that the estimators obtained here do not rely on the specific source vector (12), and are valid for any type of source vector (e.g. computed or experimentally measured).

\subsection{Misspecified MLE}

Frequently, the model used for the estimation does not match the actual physics of the problem exactly. This is the case for formulation I, which neglects the geometrical decay of radiating soundwaves. In the conditional model, by modeling the measurements as random variables following the distribution $C \mathcal{N}\left(B \mathbf{b}(\mathbf{x}), \sigma^{2} \mathbf{I}\right)$, where $b_{n}(\mathbf{x})=a_{n}(\mathbf{x}) /\left|a_{n}(\mathbf{x})\right|$ and $B$ is the complex amplitude, one shows that the parameters $\hat{\mathbf{x}}$ and $B$ maximizing the misspecified likelihood are given by using (35) and (37), replacing $\mathbf{h}^{\mathrm{IV}}$ and $\mathbf{h}^{\mathrm{III}}$ with $\mathbf{h}^{\mathrm{I}}$.

Likewise, in the unconditional model, measurements are assumed to follow the distribution $C \mathcal{N}\left(0, q \mathbf{b}(\mathbf{x}) \mathbf{b}(\mathbf{x})^{H}+\sigma^{2} \mathbf{I}\right)$, with $q$ the power, and estimation is obtained by using (39) and (42) with the same modifications as in the conditional case.

In general, the MMLE is not guaranteed to be unbiased. Nevertheless, the asymptotic mean of the MMLE is known, as it is the parameters minimizing the Kullback-Leibler divergence (KLD) [32] between the true distribution of the data and the misspecified model[30]. We recall that the KLD between two distributions with density functions $f$ and $g$ defined on $\mathbf{R}$ is given by

$$
D_{\mathrm{KL}}(f \| g)=\int_{\mathbf{R}} f(x) \log \frac{f(x)}{g(x)} \mathrm{d} x .
$$

Here, the distributions of the data and of the assumed model are complex normal, and the KLD between two complex normal distributions of dimension $N$ is given by

$$
\begin{aligned}
& D_{\mathrm{KL}}\left(C \mathcal{N}\left(\mu_{1}, \Sigma_{1}\right) \| C \mathcal{N}\left(\mu_{2}, \Sigma_{2}\right)\right) \\
& =\operatorname{tr}\left(\Sigma_{2}^{-1} \Sigma_{1}\right)+\left(\mu_{2}-\mu_{1}\right)^{H} \Sigma_{2}^{-1}\left(\mu_{2}-\mu_{1}\right)-N+\log \frac{\operatorname{det} \Sigma_{2}}{\operatorname{det} \Sigma_{1}}
\end{aligned}
$$

This expression is similar to the real normal case [32], and derived in a similar way using the probability density function (2).

In the conditional model, the covariance matrices of the two complex normal distribution are equal, and the KLD between the data and the model simplifies to

$$
D_{\mathrm{KL}}=\frac{1}{\sigma^{2}}\left\|A \mathbf{a}\left(\mathbf{x}_{s}\right)-B \mathbf{b}(\mathbf{x})\right\|_{2}^{2} .
$$

Minimization of the KLD is similar to the optimization problem encountered when deriving the MLE for the conditional model. The KLD attains its minimum when

$$
\begin{aligned}
\mathbf{x} & =\underset{\mathbf{x} \in \Omega}{\operatorname{argmax}}\left|\frac{\left\langle\mathbf{a}\left(\mathbf{x}_{s}\right), \mathbf{b}(\mathbf{x})\right\rangle}{\|\mathbf{b}(\mathbf{x})\|_{2}}\right|^{2} \\
& =\underset{\mathbf{x} \in \Omega}{\operatorname{argmax}} \operatorname{Re} \sum_{n=1}^{N} a_{n}\left(\mathbf{x}_{s}\right) \frac{\bar{a}_{n}(\mathbf{x})}{\left|a_{n}(\mathbf{x})\right|},
\end{aligned}
$$

that is when $a_{n}\left(\mathbf{x}_{s}\right)$ and $a_{n}(\mathbf{x})$ have the same angle for all $n$, yielding $\mathbf{x}=\mathbf{x}_{s}$ and showing that estimation of the position with formulation I is asymptotically unbiased. The amplitude $B$ minimizing the Kullback-Leibler divergence is given by

$$
\begin{aligned}
B & =\frac{\left\langle A \mathbf{a}\left(\mathbf{x}_{s}\right), \mathbf{b}\left(\mathbf{x}_{s}\right)\right\rangle}{\left\|\mathbf{b}\left(\mathbf{x}_{s}\right)\right\|_{2}^{2}} \\
& =\left(\frac{1}{N} \sum_{n=1}^{N} \frac{r_{0}}{r_{n}}\right) A,
\end{aligned}
$$

confirming the bias of the amplitude estimation found in section 3 , now in a case where position and amplitude are estimated jointly. Similar conclusions are reached for the unconditional model.

\section{Amplitude and power estimation for unknown position}

We now consider amplitude estimation when the source position is unknown. Using the same settings as in section 4, bias, variance, and MSE of the absolute value of the estimated amplitude at the reference point and at a fixed distance of the source are plotted using numerical simulations. In addition to the four formulations, performances for the MLE, combining formulations IV for localization and III for amplitude estimation, are given. Results for the estimation of the amplitude at the reference point $\mathbf{x}_{0}$ are given on Fig. 8. The MLE is unbiased and has the lowest MSE. Although formulations II and III are unbiased in the known position case, they are here slightly biased towards higher amplitudes. Indeed, as visible on Fig. 3, the estimated amplitude at the actual position being unbiased, the estimated amplitude at the maximum of the criterion of formulation II and III is necessarily positively biased.

Performances for the estimation of the amplitude at a fixed arbitrary distance to the source (here, $r_{\star}=1 \mathrm{~m}$ ) are given on Fig. 9. This estimated amplitude $\hat{A}_{\star}$ is obtained by

$$
\hat{A}_{\star}=\frac{\left\|\mathbf{x}_{0}-\hat{\mathbf{x}}_{s}\right\|_{2}}{r_{\star}} \hat{A}
$$

and involves both the estimated amplitude $\hat{A}$ at the reference point $\mathbf{x}_{0}$, and the estimated position $\hat{\mathbf{x}}_{s}$. For clarity, formulations I and IV, which are known to be biased even for known source position, are not considered. Compared to the previous case, variances of the estimations (in particular for the MLE) decrease with the frequency. This decay is expected, as the estimated amplitude is here also affected by errors in the estimated position (see Eq. (49)), which are decaying with increasing frequency, as is visible on figure 4. Formulations II and III are here biased towards lower amplitudes for reference point $\mathbf{x}_{0}$, as the estimated position is biased towards the array. Conversely, amplitudes are overestimated for reference point $\mathbf{x}_{0}^{\prime}$. As expected, MLE estimation of the amplitude (combination of formulations IV and III, where estimation of the position is unbiased and does not depend the reference point) offers the best performances, with unbiased estimation and minimal MSE. 

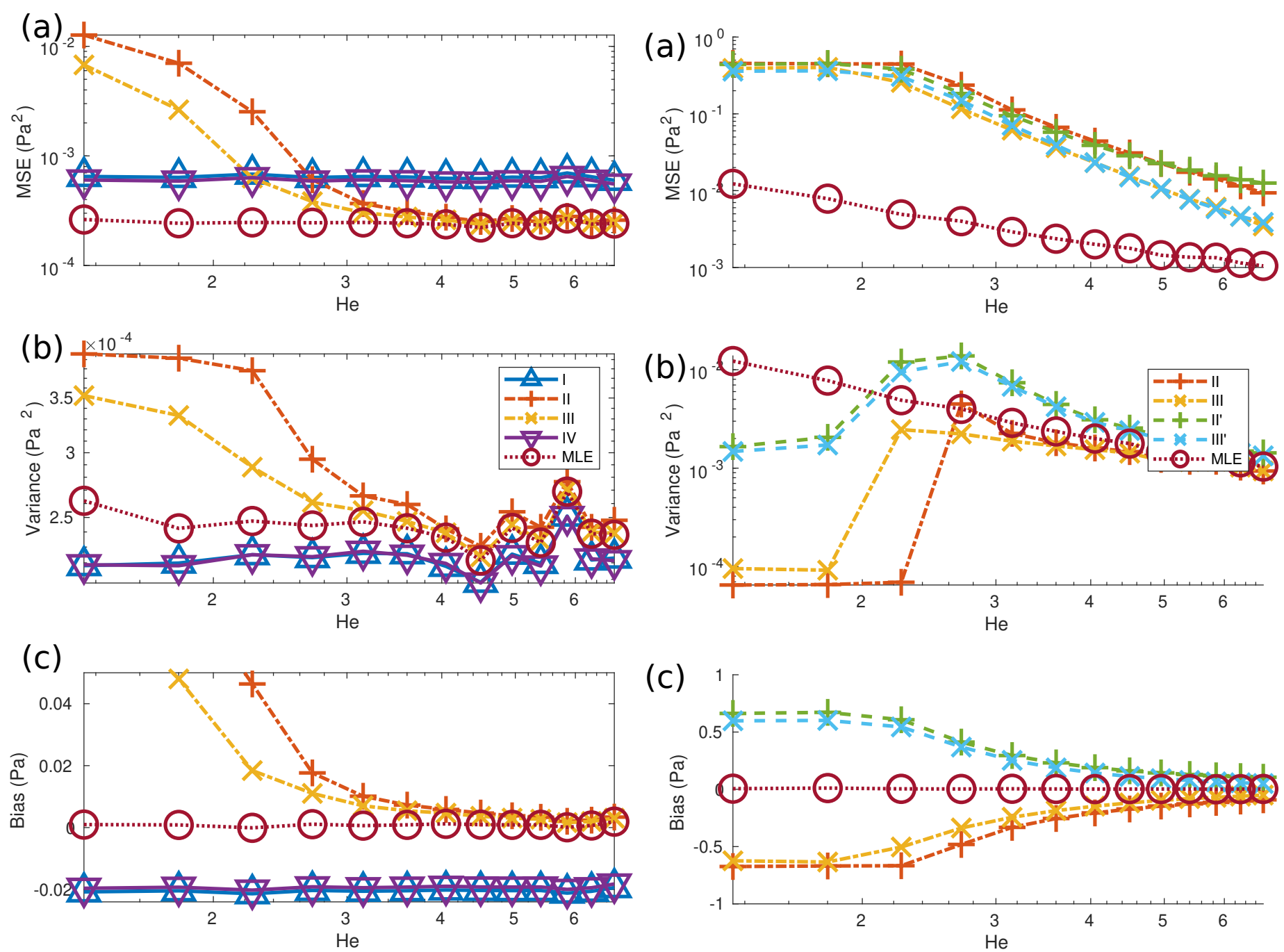

Figure 8: (a) MSE, (b) variance and (c) bias of the estimated amplitude at the reference point $\mathbf{x}_{0}$
Figure 9: (a) MSE, (b) variance and (c) bias of the estimated amplitude at a distance of $1 \mathrm{~m}$ to the source. 


\section{Non-Gaussian source and noise}

The previous results were obtained under the assumption that the source and the noise followed a Gaussian model. While this model is common, it cannot be considered to hold in all circumstances. In that case, one has to find the MLE for the specific model considered, unlikely to be identical to the MLE for Gaussian models, i.e. beamforming.

Nevertheless, some of the above results can be extended to beamforming applied to non-Gaussian models, assuming that the measurements are affected by an additive centered noise $\mathbf{n}$ with covariance matrix $\sigma^{2} \mathbf{I}$.

Elementary computations show that in the conditional model with known position, the mean and variance found in Theorem 1 still hold: the estimated amplitude $\hat{A}$ has mean $\mathrm{E}(\hat{A})=$ $A\left\langle\mathbf{a}\left(\mathbf{x}_{s}\right), \mathbf{h}\left(\mathbf{x}_{s}\right)\right\rangle$ and variance $\operatorname{Var}(\hat{A})=\sigma^{2}\left\|\mathbf{h}\left(\mathbf{x}_{s}\right)\right\|_{2}^{2}$ (however, the probability distribution of $\hat{A}$ is in in general not Gaussian). In the conditional model, the mean of the estimated power is $\mathrm{E}(\hat{p})=\left|\left\langle\mathbf{a}\left(\mathbf{x}_{s}\right), \mathbf{h}\left(\mathbf{x}_{s}\right)\right\rangle\right|^{2}$, as in Eq. (21). (the variance given in Eq. (22) is in general not valid).

Estimation of the position and amplitude of a source using formulations III and IV is in general asymptotically unbiased, for the conditional and unconditional models. This is shown by interpreting beamforming as a mismatched MLE, where the model is assumed to be Gaussian. Indeed, the KLD between a fixed arbitrary probability distribution with mean $\mu$ and covariance matrix $\Sigma$ and a multivariate normal distribution $C \mathcal{N}(\mathbf{z}, \mathbf{S})$ is minimized when $\mathbf{z}=\mu$ and $\mathbf{S}=\Sigma$. For the conditional model, with a non-Gaussian model of mean $A \mathbf{a}\left(\mathbf{x}_{s}\right)$, the Gaussian distribution minimizing the KLD has identical mean, and therefore the asymptotic mean of the estimated position and amplitude by beamforming is $\mathbf{x}_{s}$ and $A$ respectively. Likewise for the unconditional model, where the Gaussian distribution minimizing the KLD has $\Sigma=\rho \mathbf{a}\left(\mathbf{x}_{s}\right) \mathbf{a}\left(\mathbf{x}_{s}\right)^{H}+\sigma^{2} \mathbf{I}$ as covariance matrix.

\section{Conclusion}

Four beamforming steering vectors formulations were considered, extending the results of Ref. [13]. Bias and variance of position and power estimation were analyzed in the case of noisy measurements. In contrast to the far-field case, no steering vector formulation offers unbiased position and amplitude or power estimation. However, far-field source localization is a particular case where the coefficients of the source vector $\mathbf{a}(\alpha)$ have constant amplitudes, and the four formulations coincide. As this is not the case for nearfield localization, it is no surprise that accurate estimation of the position and amplitude or power necessitates more complex methods.

Formulations I and II are to be avoided. While formulation I was shown to be asymptotically unbiased for the estimation of the position, its MSE was shown to be larger than formulation IV, also unbiased, as it does not take into account the amplitude of the measured signals. Likewise, assuming that the position of the source is known, formulation II is unbiased for amplitude and power estimation. However, the unbiased formulation III was shown to have a lower variance.
Estimation of the position with formulations II and III was already known to be biased. In addition, it was shown that this bias depends on the shape of the array, the reference point, and the position of the source. Moreover, this position bias implies a corresponding bias in the estimation of the amplitude of the source in the case where the position is to be estimated.

Accurate estimation of both position and amplitude of an acoustical source necessitates the combination of formulations IV and III, for the estimation of the position and of the amplitude respectively. As it is the MLE, it is guaranteed to be asymptotically unbiased and efficient, as the SNR or number of snapshots increase. A similar procedure was used in [19], however using the less efficient formulations I and II. As it does not depend on the specific shape of the Green function, this analysis remains relevant for more general cases (e.g. inhomogeneous medium, reflections, etc., with computed or experimental source vectors). The gain in performances were even more evident for the estimation of the amplitude at an arbitrary distance to the source, necessary to predict the effect of a source at an arbitrary point in space.

This issue of the diagonal of the covariance matrix $\Sigma$ was here avoided, as the covariance matrix of the noise was assumed to be perfectly known. This issue will be considered for further research on this topic. Consequences of these results on the localization of multiple sources will also be explored.

\section{Appendix A. Bias and variance of power estimation}

In the unconditional model, the power of the source at $\mathbf{x}_{s}$ is estimated by

$$
\begin{aligned}
\hat{p} & =\mathbf{h}\left(\mathbf{x}_{s}\right)^{H}\left(\hat{\Sigma}-\sigma^{2} \mathbf{I}\right) \mathbf{h}\left(\mathbf{x}_{s}\right) \\
& =\mathbf{h}\left(\mathbf{x}_{s}\right)^{H} \hat{\Sigma} \mathbf{h}\left(\mathbf{x}_{s}\right)-\sigma^{2}\left\|\mathbf{h}\left(\mathbf{x}_{s}\right)\right\|_{2}^{2} \\
& =\frac{1}{S} \sum_{s=1}^{S}\left|\mathbf{h}\left(\mathbf{x}_{s}\right)^{H} \mathbf{m}_{s}\right|^{2}-\sigma^{2}\left\|\mathbf{h}\left(\mathbf{x}_{s}\right)\right\|_{2}^{2},
\end{aligned}
$$

using the definition of $\hat{\Sigma}(9)$. As the $\mathbf{m}_{s}$ are complex normal with covariance matrix $\Sigma$, the $\mathbf{h}\left(\mathbf{x}_{s}\right)^{H} \mathbf{m}_{s}$ are independent and identically distributed complex centered normal variables of variance $\rho=\mathbf{h}\left(\mathbf{x}_{s}\right)^{H} \Sigma \mathbf{h}\left(\mathbf{x}_{s}\right)=p\left|\mathbf{h}\left(\mathbf{x}_{s}\right)^{H} \mathbf{a}\left(\mathbf{x}_{s}\right)\right|^{2}+\sigma^{2}\left\|\mathbf{h}\left(\mathbf{x}_{s}\right)\right\|_{2}^{2}$, and $\frac{2}{\rho} \sum_{s=1}^{S}\left|\mathbf{h}\left(\mathbf{x}_{S}\right)^{H} \mathbf{m}_{s}\right|^{2}$ follows the $\chi^{2}(2 S)$ distribution, with mean $2 S$ and variance $4 S$ (it can be seen as the sum of squares of the real and imaginary part of the $\mathbf{h}\left(\mathbf{x}_{s}\right)^{H} \mathbf{m}_{s}$, i.e. $2 S$ independent and identically distributed real centered normal variables of variance $\rho / 2$ ). The first term of (A.3) has mean $\rho$ and variance $\rho^{2} / S$, and the second term is deterministic, yielding (21) and (22).

\section{Appendix B. Position bias of formulations II and III}

For a source at $\mathbf{x}_{s}$, we define $b_{\mathrm{II}}(s)=\left\langle\mathbf{a}\left(\mathbf{x}_{s}\right), \mathbf{h}^{\mathrm{II}}\left(\mathbf{x}_{s}+s \mathbf{u}\right)\right\rangle$. Then,

$$
\begin{aligned}
& B_{\mathrm{II}}(s)=\left|b_{\mathrm{II}}(s)\right|^{2} \\
& B_{\mathrm{II}}^{\prime}(s)=2 \operatorname{Re}\left(\bar{b}_{\mathrm{II}}(s) b_{\mathrm{II}}^{\prime}(s)\right) \\
& B_{\mathrm{II}}^{\prime \prime}(s)=2 \operatorname{Re}\left(\bar{b}_{\mathrm{II}}(s) b_{\mathrm{II}}^{\prime \prime}(s)+\left|b_{\mathrm{II}}^{\prime}(s)\right|^{2}\right)
\end{aligned}
$$


From the definition of $\mathbf{h}^{\mathrm{II}}$,

$$
b_{\mathrm{II}}(s)=\frac{1}{N} \sum_{n=1}^{N} \frac{r_{n}(s) r_{0}(0)}{r_{0}(s) r_{n}(0)} \mathrm{e}^{\mathrm{i} k\left(r_{n}(s)-r_{0}(s)\right)} \mathrm{e}^{-\mathrm{i} k\left(r_{n}(0)-r_{0}(0)\right)}
$$

Routine computations yield

$$
\begin{aligned}
& b_{\mathrm{II}}(0)=1 \\
& b_{\mathrm{II}}^{\prime}(0)=\frac{1}{N} \sum_{n=1}^{N}\left(\frac{r_{n}^{\prime}}{r_{n}}-\frac{r_{0}^{\prime}}{r_{0}}+\mathrm{i} k\left(r_{n}^{\prime}-r_{0}^{\prime}\right)\right) \\
& b_{\mathrm{II}}^{\prime \prime}(0)=\frac{1}{N} \sum_{n=1}^{N}\left(-k^{2}\right)\left(r_{n}^{\prime}-r_{0}^{\prime}\right)^{2}+2 \mathrm{i} k\left(\left(r_{n}^{\prime}-r_{0}^{\prime}\right)\left(\frac{r_{n}^{\prime}}{r_{n}}-\frac{r_{0}^{\prime}}{r_{0}}\right)+\left(r_{n}^{\prime \prime}-r_{0}^{\prime \prime}\right)\right. \\
& +2 \frac{r_{0}^{\prime}}{r_{0}}\left(\frac{r_{0}^{\prime}}{r_{0}}-\frac{r_{n}^{\prime}}{r_{n}}\right)+\frac{r_{n}^{\prime \prime}}{r_{n}}-\frac{r_{0}^{\prime \prime}}{r_{0}} \\
& B_{\mathrm{II}}^{\prime}(0)=\frac{2}{N} \sum_{n=1}^{N}\left(\frac{r_{n}^{\prime}}{r_{n}}-\frac{r_{0}^{\prime}}{r_{0}}\right) \\
& B_{\mathrm{II}}^{\prime \prime}(0) \approx \frac{2}{N}\left(\sum_{n=1}^{N}\left(-k^{2}\right)\left(r_{n}^{\prime}-r_{0}^{\prime}\right)^{2}+\frac{k^{2}}{N}\left(\sum_{n=1}^{N}\left(r_{n}^{\prime}-r_{0}^{\prime}\right)\right)^{2}\right) \\
& =-\frac{2 k^{2}}{N}\left(\sum_{n=1}^{N}\left(r_{n}^{\prime}\right)^{2}-\frac{1}{N}\left(\sum_{n=1}^{N} r_{n}^{\prime}\right)^{2}\right)
\end{aligned}
$$

yielding (31).

Bias in null when $B_{\mathrm{II}}^{\prime}(0)=0$. This is obtained in the particular case where the source is located at $(0,0, Z)$, searched on the $z$ axis, and the microphones and reference point are placed on the sphere of center $(0,0, Z / 2)$ and diameter $Z / 2$. Indeed, in this case, $r_{0}(s)=Z+s$, and for a microphone at position $n$, $r_{n}(s)=\sqrt{x_{n}^{2}+y_{n}^{2}+\left(s+Z-z_{n}\right)^{2}}$. Then,

$$
\frac{r_{n}^{\prime}(0)}{r_{n}(0)}=\frac{Z-z_{n}}{x_{n}^{2}+y_{n}^{2}+\left(Z-z_{n}\right)^{2}}
$$

and combined with the equation of the sphere $x^{2}+y^{2}+(z-$ $Z / 2)^{2}=Z^{2} / 4, \frac{r_{n}^{\prime}(0)}{r_{n}(0)}=1 / Z$. As the reference point is also on the sphere, $\frac{r_{0}^{\prime}(0)}{r_{0}(0)}=1 / Z$, and $B_{\mathrm{II}}^{\prime}(0)=0$.

For formulation III, similar computations yield

$$
\begin{aligned}
& b_{\mathrm{III}}(0)=1 \\
& b_{\mathrm{III}}^{\prime}(0)=\frac{1}{\sum_{n=1}^{N} \frac{1}{r_{n}^{2}}} \sum_{n=1}^{N} \frac{1}{r_{n}^{2}}\left(\frac{r_{n}^{\prime}}{r_{n}}-\frac{r_{0}^{\prime}}{r_{0}}+\mathrm{i} k r_{n}^{\prime}-\mathrm{i} k r_{0}^{\prime}\right) \\
& b_{\mathrm{III}}^{\prime \prime}(0) \approx-k^{2} \frac{1}{\sum_{n=1}^{N} \frac{1}{r_{n}^{2}}} \sum_{n=1}^{N} \frac{1}{r_{n}^{2}}\left(r_{n}^{\prime}-r_{0}^{\prime}\right)^{2} .
\end{aligned}
$$

Compared to formulation II, the terms of the sums are weighted by $1 / r_{n}^{2}$.

\section{Appendix C. Maximum likelihood estimators}

\section{Appendix C.1. Conditional model}

As $\mathbf{m}$ is assumed to follow the distribution $C \mathcal{N}\left(A \mathbf{a}(\mathbf{x}), \sigma^{2} \mathbf{I}\right)$ parameterized by the position $\mathbf{x}$ and the amplitude $A$, its probability density is given by

$$
f_{(\mathbf{x}, A)}(\mathbf{m})=\frac{1}{\pi^{N} \sigma^{2 N}} \exp \left(-\frac{\|\mathbf{m}-A \mathbf{a}(\mathbf{x})\|_{2}^{2}}{\sigma^{2}}\right)
$$

Given the data $\mathbf{m}$, the parameters $\mathbf{x}$ and $A$ are estimated by maximizing the likelihood $l_{\mathbf{m}}(\mathbf{x}, A)=f_{(\mathbf{x}, A)}(\mathbf{m})$, or, equivalently, ) polving the minimization problem

$$
(\hat{\mathbf{x}}, \hat{A})=\underset{\mathbf{x} \in \Omega, A \in \mathbf{C}}{\operatorname{argmin}}\|\mathbf{m}-A \mathbf{a}(\mathbf{x})\|_{2}^{2} .
$$

By first solving the problem for $A$ with fixed position $\mathbf{x}$, and then solving for $\mathbf{x}$, solution of this optimization problem is given by

$$
\begin{aligned}
& \hat{\mathbf{x}}=\underset{\mathbf{x} \in \Omega}{\operatorname{argmax}}\left|\left\langle\mathbf{m}, \frac{\mathbf{a}(\mathbf{x})}{\|\mathbf{a}(\mathbf{x})\|_{2}}\right\rangle\right|^{2} \\
& \hat{A}=\left\langle\mathbf{m}, \frac{\mathbf{a}(\mathbf{x})}{\|\mathbf{a}(\mathbf{x})\|_{2}^{2}}\right\rangle .
\end{aligned}
$$

\section{Appendix C.2. Unconditional model}

In the unconditional model, several snapshots $\mathbf{m}_{s}$ are collected. They are assumed to be independent, and to follow the distribution $\operatorname{CN}\left(0, \Sigma_{p, \mathbf{x}}\right)$, with $\Sigma_{p, \mathbf{x}}=p \mathbf{a}(\mathbf{x}) \mathbf{a}(\mathbf{x})^{H}+\sigma^{2} \mathbf{I}$, and joint density

$$
f_{\mathbf{x}, p}\left(\mathbf{m}_{1}, \ldots, \mathbf{m}_{S}\right)=\prod_{s=1}^{S} \frac{1}{\pi^{N} \operatorname{det}\left(\Sigma_{p, \mathbf{x}}\right)} \exp \left(-\mathbf{m}_{s} \Sigma_{p, \mathbf{x}}^{-1} \mathbf{m}_{s}\right)
$$

The log-likelihood is given by

$$
\begin{aligned}
L(\mathbf{x}, p) & =\log f_{\mathbf{x}, p}\left(\mathbf{m}_{1}, \ldots, \mathbf{m}_{S}\right) \\
& =-\sum_{s=1}^{S} \mathbf{m}_{s} \Sigma_{p, \mathbf{x}}^{-1} \mathbf{m}_{s}-S \log \operatorname{det}\left(\Sigma_{p, \mathbf{x}}\right)-N S \log \pi \\
& =-S \operatorname{tr}\left(\Sigma_{p, \mathbf{x}}^{-1} \hat{\Sigma}\right)-S \log \operatorname{det}\left(\Sigma_{p, \mathbf{x}}\right)-N S \log \pi
\end{aligned}
$$

using the definition (9) of $\hat{\Sigma}$ and the properties of the trace. Maximizing the likelihood is thus equivalent to solving the optimization problem

$$
(\hat{\mathbf{x}}, \hat{p})=\underset{\mathbf{x} \in \Omega, p \in \mathbf{R}_{+}}{\operatorname{argmin}} \operatorname{tr}\left(\Sigma_{p, \mathbf{x}}^{-1} \hat{\Sigma}\right)+\log \operatorname{det}\left(\Sigma_{p, \mathbf{x}}\right)
$$

By the matrix determinant lemma and the Sherman-Morrison formula,

$$
\begin{aligned}
\operatorname{det}\left(\Sigma_{p, \mathbf{x}}\right) & =\sigma^{2(N-1)}\left(\sigma^{2}+p\|\mathbf{a}(\mathbf{x})\|^{2}\right) \\
\Sigma_{p, \mathbf{x}}^{-1} & =\frac{1}{\sigma^{2}}\left(I-\frac{p \mathbf{a}(\mathbf{x}) \mathbf{a}(\mathbf{x})^{H}}{\sigma^{2}+p\|\mathbf{a}(\mathbf{x})\|^{2}}\right)
\end{aligned}
$$


and

$$
(\hat{\mathbf{x}}, \hat{p})=\underset{\mathbf{x} \in \Omega, p \in \mathbf{R}_{+}}{\operatorname{argmin}} \frac{\operatorname{tr} \hat{\Sigma}}{\sigma^{2}}-\frac{p \mathbf{a}(\mathbf{x})^{H} \hat{\Sigma} \mathbf{a}(\mathbf{x})}{\sigma^{2}\left(\sigma^{2}+p\|\mathbf{a}(\mathbf{x})\|^{2}\right)}+\log \left(\sigma^{2}+p\|\mathbf{a}(\mathbf{x})\|^{2}\right)
$$

For fixed $\mathbf{x}$, the optimal value of $p$ is given by

$$
p^{\star}(\mathbf{x})=\frac{1}{\|\mathbf{a}(\mathbf{x})\|^{2}}\left(\frac{\mathbf{a}(\mathbf{x})^{H} \hat{\Sigma} \mathbf{a}(\mathbf{x})}{\|\mathbf{a}(\mathbf{x})\|^{2}}-\sigma^{2}\right) .
$$

Injecting in (C.12) and removing constant terms with respect to $\mathbf{x}$, the position is found by solving

$$
\hat{\mathbf{x}}=\underset{\mathbf{x} \in \Omega}{\operatorname{argmax}} \frac{1}{\sigma^{2}} \frac{\mathbf{a}(\mathbf{x})^{H} \hat{\Sigma} \mathbf{a}(\mathbf{x})}{\|\mathbf{a}(\mathbf{x})\|^{2}}-\log \frac{\mathbf{a}(\mathbf{x})^{H} \hat{\Sigma} \mathbf{a}(\mathbf{x})}{\|\mathbf{a}(\mathbf{x})\|^{2}},
$$

and the estimated power $\hat{p}$ is finally given by $p^{\star}(\hat{\mathbf{x}})$.

\section{Appendix C.3. Non-Gaussian models}

We show here that with $p$ a probability density with mean $\mu$ and covariance matrix $\Sigma$, the complex Gaussian distribution $q$ minimizing the $\operatorname{KLD} D_{\mathrm{KL}}(p \| q)$ is $C \mathcal{N}(\mu, \Sigma)$.

The KLD between $p$ and $q$ is

$$
\begin{aligned}
D_{\mathrm{KL}}(p \| q) & =\int_{\mathbf{C}^{N}} p(\mathbf{x}) \log \frac{p(\mathbf{x})}{q(\mathbf{x})} \mathrm{d} \mathbf{x} \\
& =\int_{\mathbf{C}^{N}} p(\mathbf{x}) \log p(\mathbf{x})-p(\mathbf{x}) \log q(\mathbf{x}) \mathrm{d} \mathbf{x} .
\end{aligned}
$$

The first term is the differential entropy of $p$, constant with respect to the mean and covariance of $q$. With $\mathbf{z}$ and $\mathbf{S}$ the mean and covariance of $q$, using Eq. (2), the second term writes

$$
\begin{aligned}
& -\int_{\mathbf{C}^{N}} p(\mathbf{x}) \log q(\mathbf{x}) \mathrm{d} \mathbf{x} \\
& =-\int_{\mathbf{C}^{N}} p(\mathbf{x})\left(-N \pi-\log \operatorname{det} \mathbf{S}-(\mathbf{x}-\mathbf{z})^{H} \mathbf{S}^{-1}(\mathbf{x}-\mathbf{z})\right) \mathrm{d} \mathbf{x} \\
& =N \pi+\log \operatorname{det} \mathbf{S}+\operatorname{tr}\left(\mathbf{S}^{-1} \int_{\mathbf{C}^{N}} p(\mathbf{x})(\mathbf{x}-\mathbf{z})(\mathbf{x}-\mathbf{z})^{H} \mathrm{~d} \mathbf{x}\right) \\
& =N \pi+\log \operatorname{det} \mathbf{S}+\operatorname{tr}\left(\mathbf{S}^{-1}\left(\Sigma+(\mu-\mathbf{z})(\mu-\mathbf{z})^{H}\right)\right) \\
& =N \pi+\log \operatorname{det} \mathbf{S}+\operatorname{tr}\left(\mathbf{S}^{-1} \Sigma\right)+(\mu-\mathbf{z})^{H} \mathbf{S}^{-1}(\mu-\mathbf{z})
\end{aligned}
$$

With fixed $\mathbf{S}$, the KLD is minimized when $\mathbf{z}=\mu$. Then, minimizing $\log \operatorname{det} \mathbf{S}+\operatorname{tr}\left(\mathbf{S}^{-1} \Sigma\right)$ yields $\mathbf{S}=\Sigma$.

\section{References}

[1] R. Merino-Martínez, P. Sijtsma, M. Snellen, T. Ahlefeldt, J. Antoni, C. J. Bahr, D. Blacodon, D. Ernst, A. Finez, S. Funke, T. F. Geyer, S. Haxter, G. Herold, X. Huang, W. M. Humphreys, Q. Leclère, A. Malgoezar, U. Michel, T. Padois, A. Pereira, C. Picard, E. Sarradj, H. Siller, D. G. Simons, C. Spehr, A review of acoustic imaging methods using phased microphone arrays: Part of the "Aircraft Noise Generation and Assessment" Special Issue, CEAS Aeronaut. J. 10 (1) (2019) 197-230. doi:10.1007/s13272-019-00383-4.

[2] R. Schmidt, Multiple emitter location and signal parameter estimation, IEEE Trans. on Antennas Propag. 34 (3) (1986) 276-280. doi:10.1109/TAP.1986.1143830.
[3] R. Roy, T. Kailath, ESPRIT-estimation of signal parameters via rotational invariance techniques, IEEE Trans. on Acoust. Speech, Signal Process. 37 (7) (1989) 984-995. doi:10.1109/29.32276.

[4] T. Padois, A. Berry, Orthogonal matching pursuit applied to the deconvolution approach for the mapping of acoustic sources inverse problem, The J. Acoust. Soc. Am. 138 (6) (2015) 3678-3685. doi:10.1121/1.4937609.

[5] P. Simard, J. Antoni, Acoustic source identification: Experimenting the $\ell_{1}$ minimization approach, Appl. Acoust. 74 (7) (2013) 974-986. doi:10.1016/j.apacoust.2013.01.012.

[6] E. Sarradj, A fast signal subspace approach for the determination of absolute levels from phased microphone array measurements, J. Sound Vib. 329 (9) (2010) 1553-1569. doi:10.1016/j.jsv.2009.11.009.

[7] P. Sijtsma, R. Merino-Martinez, A. M. Malgoezar, M. Snellen, High-resolution CLEAN-SC: Theory and experimental validation, Int. J. Aeroacoustics $16 \quad$ (4-5) (2017) 274-298. doi:10.1177/1475472X17713034.

[8] T. F. Brooks, W. M. Humphreys, A deconvolution approach for the mapping of acoustic sources (DAMAS) determined from phased microphone arrays, J. Sound Vib. 294 (4) (2006) 856-879. doi:10.1016/j.jsv.2005.12.046.

[9] T. Yardibi, J. Li, P. Stoica, L. N. Cattafesta, Sparsity constrained deconvolution approaches for acoustic source mapping, The J. Acoust. Soc. Am. 123 (5) (2008) 2631-2642. doi:10.1121/1.2896754.

[10] G. Chardon, J. Picheral, F. Ollivier, Theoretical analysis of the DAMAS algorithm and efficient implementation of the covariance matrix fitting method for large-scale problems, J. Sound Vib. 508 (2021) 116208. doi:10.1016/j.jsv.2021.116208.

[11] P. Stoica, A. Nehorai, Performance study of conditional and unconditional direction-of-arrival estimation, IEEE Trans. on Acoust. Speech, Signal Process. 38 (10) (1990) 1783-1795. doi:10.1109/29.60109.

[12] G. Herold, E. Sarradj, Performance analysis of microphone array methods, J. Sound Vib. 401 (2017) 152-168. doi:10.1016/j.jsv.2017.04.030.

[13] E. Sarradj, Three-Dimensional Acoustic Source Mapping with Different Beamforming Steering Vector Formulations, Adv. Acoust. Vib. 2012 (2012) e292695, publisher: Hindawi. doi:10.1155/2012/292695.

[14] G. Chardon, gilleschardon/beamformingtheory, doi:10.5281/zenodo.5119269 https://doi.org/10.5281/zenodo.5519269 last accessed 21/09/2021.

[15] B. Picinbono, Second-order complex random vectors and normal distributions, IEEE Trans. on Signal Process. 44 (10) (1996) 2637-2640. doi:10.1109/78.539051.

[16] T. Padois, A. Berry, Two and Three-Dimensional Sound Source Localization with Beamforming and Several Deconvolution Techniques, Acta Acustica united with Acustica 103 (2017) 10.

[17] D. Sun, C. Ma, J. Mei, W. Shi, Improving the resolution of underwater acoustic image measurement by deconvolution, Appl. Acoust. 165 (2020) 107292. doi:https://doi.org/10.1016/j.apacoust.2020.107292.

[18] H. Ding, Y. Bao, Q. Huang, C. Li, G. Chai, Three-dimensional localization of point acoustic sources using a planar microphone array combined with beamforming, Royal Soc. Open Sci. 5 (12) (2018). doi:10.1098/rsos.181407.

[19] M. Polichetti, V. Baron, J. I. Mars, B. Nicolas, Multiplane deconvolution in underwater acoustics: Simultaneous estimations of source level and position, JASA Express Lett. 1 (7) (2021) 076001. doi:10.1121/10.0005513.

[20] L. Chen, Y. Xiao, T. Yang, Application of the improved fast iterative shrinkage-thresholding algorithms in sound source localization, Appl. Acoust. $180 \quad$ (2021) 108101. doi:https://doi.org/10.1016/j.apacoust.2021.108101.

[21] L. Gilquin, S. Bouley, J. Antoni, T. Le Magueresse, C. Marteau, Sensitivity analysis of two inverse methods: Conventional Beamforming and Bayesian focusing, J. Sound Vib. 455 (2019) 188-202. doi:10.1016/j.jsv.2019.05.002.

[22] H.-G. Raumer, C. Spehr, T. Hohage, D. Ernst, Weighted data spaces for correlation-based array imaging in experimental aeroacoustics, J. Sound Vib. 494 (2021) 115878. doi:10.1016/j.jsv.2020.115878.

[23] Y. Zhou, V. Valeau, J. Marchal, F. Ollivier, R. Marchiano, Threedimensional identification of flow-induced noise sources with a tunnelshaped array of MEMS microphones, J. Sound Vib. 482 (2020) 115459. doi:10.1016/j.jsv.2020.115459.

[24] W. Ma, H. Bao, C. Zhang, X. Liu, Beamforming of phased microphone array for rotating sound source localization, J. Sound Vib. 467 (2020) 
115064. doi:10.1016/j.jsv.2019.115064.

[25] X. Wang, B. Quost, J.-D. Chazot, J. Antoni, Iterative beamforming for identification of multiple broadband sound sources, J. Sound Vib. 365 (2016) 260-275. doi:10.1016/j.jsv.2015.11.036.

[26] T. Padois, J. Fischer, C. Doolan, O. Doutres, Acoustic imaging with conventional frequency domain beamforming and generalized cross correlation: a comparison study, Appl. Acoust. 177 (2021) 107914. doi:10.1016/j.apacoust.2021.107914.

[27] J. Fischer, C. Doolan, Improving acoustic beamforming maps in a reverberant environment by modifying the cross-correlation matrix, J. Sound Vib. 411 (2017) 129-147. doi:10.1016/j.jsv.2017.09.006.

[28] S. Boyd, L. Vandenberghe, Convex Optimization, Cambridge University Press, USA, 2004, chapter 9.

[29] E. Lehmann, G. Casella, Theory of Point Estimation, 2nd Edition, Springer, 1998, chapter 6.

[30] S. Fortunati, F. Gini, M. S. Greco, C. D. Richmond, Performance Bounds for Parameter Estimation under Misspecified Models: Fundamental Findings and Applications, IEEE Signal Process. Mag. 34 (6) (2017) 142-157. doi:10.1109/MSP.2017.2738017.

[31] A. Renaux, P. Forster, E. Chaumette, P. Larzabal, On the high-SNR conditional maximum-likelihood estimator full statistical characterization, IEEE Trans. on Signal Process. 54 (12) (2006) 4840-4843.

[32] S. Kullback, Information Theory and Statistics, Dover, 1968, chapter 9. 